\section{Response to belimumab among patients with systemic lupus erythematosus in clinical practice settings: $24-$ month results from the OBSErve study in the USA}

\author{
C E Collins, ${ }^{1} \mathrm{M}$ Dall'Era, ${ }^{2} \mathrm{H}$ Kan, ${ }^{3} \mathrm{C}$ Macahilig, ${ }^{4} \mathrm{C}$ Molta,,${ }^{5} \mathrm{~V}$ Koscielny, ${ }^{6}$ \\ D J Chang ${ }^{5}$
}

To cite: Collins CE, Dall'Era M, Kan $\mathrm{H}$, et al. Response to belimumab among patients with systemic lupus erythematosus in clinical practice settings: 24-month results from the OBSErve study in the USA. Lupus Science \& Medicine 2016;3:e000118.

doi:10.1136/lupus-2015000118

- Additional material is available. To view please visit the journal (http://dx.doi.org/ 10.1136/lupus-2015000118).

Received 23 July 2015 Revised 5 November 2015 Accepted 17 November 2015

\section{CrossMark}

For numbered affiliations see end of article.

\section{Correspondence to} Dr Christopher E Collins; christopher.e.collins@ medstar.net

\section{ABSTRACT}

Objective: To examine disease activity and clinical outcomes, and describe overall patterns of systemic lupus erythematosus (SLE) care in patients who received belimumab in a real-world clinical setting. Methods: This observational cohort study was conducted in US clinical practices. Rheumatologists $(n=92)$ identified adults with SLE who had received $\geq 8$ infusions of belimumab plus standard of care (SoC). Physicians assessed disease outcomes at 6-month intervals using patient medical charts, for up to 24 months. The primary outcome was physicianassessed change in SLE disease. Other outcomes included change in steroid use, laboratory tests and healthcare resource utilisation (HCRU).

Results: Of 501 patients (intent-to-treat population (ITT)), 446 were female, mean age was 43.3 years and $98 \%$ had moderate/severe disease activity at baseline (first dose of belimumab). Data for 277 patients who completed 24 months of belimumab treatment were available. Among the ITT, a $\geq 50 \%$ improvement in overall clinical response between baseline and month 6 was reported for $48.7 \%$ of patients; continued improvement was seen at all subsequent 6 -month intervals relative to the previous timepoint. The percentage of patients with moderate/severe disease also decreased at each timepoint. At baseline, $77.0 \%$ of patients received steroids at a mean (SD) prednisone equivalent dose of 19.9 (14.39) mg/day, which decreased to 8.4 (7.35) $\mathrm{mg} /$ day at month 6 and 6.1 (9.31) mg/day at month 24 . Abnormal laboratory values typically associated with SLE also demonstrated improvements at month 6 , which continued through 24 months. HCRU decreased over the duration of the study.

Conclusions: Patients with SLE who received belimumab plus SoC for up to 24 months demonstrated improvements in disease severity and laboratory values and a reduction in steroid use and HCRU as early as month 6. Improvements continued through 24 months, providing evidence of reduced disease activity among patients taking belimumab in real-world clinical practice.

\section{KEY MESSAGES}

- This single-arm observational study found that patients with systemic lupus erythematosus (SLE) treated with belimumab plus standard of care experienced a reduction in disease activity, a decrease in steroid use, improvements in abnormal laboratory values associated with SLE and a reduction in health care resource utilisation.

- Clinical improvements were greatest during the first 6 months of receiving belimumab and were maintained through 24 months.

- Subgroup analyses demonstrated that patients with high disease activity and African-Americans had similar responses to belimumab as the overall population.

\section{INTRODUCTION}

Belimumab is a human immunoglobulin-G1 $\lambda$ monoclonal antibody that inhibits B-lymphocyte stimulator. ${ }^{1}$ It is indicated for the treatment of patients with active, autoantibody-positive systemic lupus erythematosus (SLE) receiving standard of care (SoC). ${ }^{2}$ Two large randomised, placebocontrolled trials, BLISS- $52^{3}$ and BLISS-76, demonstrated the efficacy and safety of belimumab plus SoC in patients with SLE. Trials such as these are essential to demonstrate efficacy and safety of a new treatment; however, strict inclusion and exclusion criteria, dosing limitations and inflexible concomitant medication management can limit the generalisability of clinical trial data into patient populations and clinical practice. ${ }^{5}$

Understanding physician-chosen patterns of treatment initiation, concomitant medication management, patient subgroup response and treatment effectiveness in a 
community-based setting may provide valuable clinical practice insights. In this study (Evaluation Of use of Belimumab in clinical practice SEttings in the US (OBSErve US)), we investigated the clinical effectiveness, changes in laboratory parameters and healthcare resource utilisation (HCRU) of belimumab plus SoC in a cohort of patients with SLE over 24 months in clinical practice.

\section{METHODS}

Study design

This observational cohort study (GSK study 117295) was conducted in US rheumatology practices between February 2012 and May 2014. Physicians were not informed of the identity of the study sponsor and participating physicians remained anonymous to the study sponsor. Patient data were anonymised.

The study was performed in two stages. Stage 1, from 6 months prior to first belimumab infusion (baseline) to 12 months post-baseline, was a retrospective review of medical chart data from patients with SLE (with the exception of 284 patients for whom month 12 data were collected prospectively). In stage 2 (months 18-24), patient follow-up was tracked prospectively. All patients completed at least 6 months post-baseline.

\section{Study physicians}

A systematic sampling method was used to randomly recruit non-academic rheumatologists from a US national physician database (Firstmark, Compton, New Hampshire, USA). From a list of 6842, every 46th physician was contacted to determine study eligibility and interest in participation. If unsuccessful, the next physician on the list was contacted. Eligible physicians had $\geq 5$ years of SLE practice experience, treated $\geq 10$ patients with SLE annually, had $\geq 5$ patients receiving marketed belimumab and had $\geq 1$ patient who had received $\geq 8$ infusions of belimumab.

\section{Study patients}

Physicians identified medical charts of patients with SLE, aged $\geq 18$ years, with $\geq 6$ months of documented medical history prior to belimumab initiation, who had received $\geq 8$ intravenous infusions of belimumab $10 \mathrm{mg} / \mathrm{kg}$ (equivalent to approximately 6 months of treatment) plus SoC and had a documented reason for belimumab initiation. Patients were excluded if they were enrolled in a SLE clinical trial while treated with belimumab. Each physician was limited to enrolling no more than 25 patients. Patients were selected at random from a list of all eligible patients from each physician's practice.

\section{Study objectives and assessments}

The primary objective was to examine disease activity, clinical outcomes and describe overall patterns of SLE care. The primary outcome was overall clinical response to belimumab at the end of each 6-month interval, relative to the beginning of the 6 -month period (categorised as worse, no improvement, $<20 \%, 20-49 \%, 50$ $79 \%, \geq 80 \%$ improvement). This was based on the physician's assessment of chart data from the patient's closest clinical visit to the start and end of each 6-month period (baseline-month 6, month 6-12, month 12-18 and month 18-24). Other clinical outcome measures included physician assessment of overall SLE disease severity (mild, moderate, severe), Safety of Estrogens in Lupus National Assessment-Systemic Lupus Erythematosus Disease Activity Index (SELENA-SLEDAI; where available), clinical manifestations and use of corticosteroids. Laboratory tests (serum creatinine, $24 \mathrm{~h}$ urine protein, spot urine protein-to-creatinine ratio, haematology, liver function, complement (C3 and C4) levels, anti-double-stranded DNA (anti-dsDNA) antibodies, serum $\mathrm{C}$ reactive protein (CRP) and erythrocyte sedimentation rate (ESR)) data collection was added as a protocol amendment (January 2014). Tests were performed at local laboratories and data (where available) were collected at baseline, months 6,12 and 24 . The test performed closest to, and before, the timepoint of interest was used. Standard ranges of normal levels were established for each test to enable comparison of categorised data.

To assess disease flare prevention and maintenance of disease control, additional post hoc analyses were conducted.

HCRU, including number of office visits, visits to other specialties for SLE-related reasons, emergency room (ER) visits/hospitalisations and ancillary care services, was a secondary outcome.

Although safety evaluations were not an objective, adverse events (AEs) that the physician deemed belimumab-related were reported to the study sponsor and those cited as a reason for belimumab discontinuation were recorded.

\section{Data collection}

Data were collected via case report forms (CRFs) completed by the treating physician (a physician practice profile form, patient CRFs for each time period (baseline6 months; see online supplementary file 1; 6-12 months, 12-18 months and 18-24 months; see online supplementary file 2 and a laboratory test CRF; see online supplementary file 3). Physicians were given detailed instructions and followed up to ensure any questions were addressed. Outcome measures were reported by physicians based on their own assessment during CRF completion or usual practice. Where data were missing or illegible, physicians were contacted to check the missing data against the patient's medical chart and, if necessary, any missing data were documented as such.

Clinical manifestations and severity at each timepoint were assessed according to the organ domains and symptoms listed in the study legend sheet (see online supplementary file 4 ). 
Data analyses

Descriptive analyses were performed for all study objectives. A sample size of 500 was chosen based on feasibility.

The intent-to-treat (ITT) population consisted of all patients identified for inclusion who completed at least 6 months; the last observation carried forward (LOCF) method was used to impute missing values after 6 months. When a patient had only baseline values, they were not carried forward and the patient was excluded from the analysis; therefore, for some analyses patient numbers are lower than the ITT population. All analyses of the ITT population were performed post hoc as the original approach was based on analysis of completers only. Baseline differences between the 24-month completers and patients who dropped out were compared in sensitivity analyses ( $\chi^{2}$ and analysis of variance).

Prednisone-equivalent doses were calculated for nonprednisone steroids. Post hoc subgroup analyses examined patients with high disease activity at baseline (anti-dsDNA-positive and low C3/C4, SELENA-SLEDAI $>10$ or steroid dose $>7.5 \mathrm{mg} /$ day) and predefined analyses examined ethnic subgroups (Caucasian, African-American, Hispanic and other). Fisher's exact tests were conducted to compare the primary end point results at month 6 and month 24 in subgroup analyses. HCRU end points were prespecified and analysed for the 24-month completer population; LOCF was not considered appropriate due to the high variability in these data.

\section{RESULTS}

Physician characteristics

A total of 92 rheumatologists from 27 states participated, with a mean (SD) duration of practice of 16.4 (8.72) years. The mean number of patients per practice was 2046; the mean number of patients with SLE was 196, of whom a mean of 11 patients were receiving belimumab. Per practice, a mean of 5.4 patients (range 1-25) contributed to the study. SELENA-SLEDAI was used by $13(14.1 \%)$ physicians, which corresponded to 122 (24.4\%) patients; $42.4 \%$ of physicians reported that they did not use any disease activity assessment tools.

\section{Patient disposition}

The ITT population consisted of 501 patients. By month 24, $112(22.4 \%)$ patients were lost to follow-up and 112 $(22.4 \%)$ discontinued belimumab treatment; 277 $(55.3 \%$ ) patients remained (figure 1). The most common reasons for discontinuation were patient request $(\mathrm{n}=45 ; 40.2 \%)$ and medication not effective $(n=33 ; 29.5 \%)$. There were 14 patients that discontinued due to AEs, with sepsis $(n=4)$ and depression $(n=3)$ most frequently reported. One patient died during the study due to central nervous system lupus that was deemed by the treating physician to be not treatment related.

\section{Baseline demographics and disease characteristics}

The majority of the ITT population were female $(n=446$; $89.0 \%)$ and Caucasian $(\mathrm{n}=265 ; 52.9 \%)$; mean (SD) age at baseline was 41.3 (12.10) years (table 1). At baseline, $2.2 \%, 77.6 \%$ and $20.2 \%$ of patients were assessed by their physician as having mild, moderate and severe SLE, respectively, and mean (SD) SELENA-SLEDAI score $(\mathrm{n}=122)$ was 12.4 (3.62). Almost all patients (97.2\%) received concomitant therapy at baseline, including oral steroids (77.0\%), antimalarials $(69.7 \%)$ and immunosuppressants $(58.9 \%)$ such as methotrexate $(23.2 \%)$, mycophenolate mofetil (19.2\%) and azathioprine (18.6\%). The most common reasons for belimumab initiation were that the previous treatment regimen was not effective $(71.3 \%)$, desire to decrease steroids (57.1\%) and worsening patient condition $(56.7 \%)$. The most common organ system manifestations were musculoskeletal $(76.9 \%)$, mucocutaneous $(63.5 \%)$, constitutional $(56.7 \%)$, immunological $(54.0 \%)$ and haematological $(35.3 \%)$.

The 24-month completer population characteristics were consistent with the ITT population (table 1). A sensitivity analysis, comparing patients who completed versus those who did not found that the only significant differences in baseline characteristics was that fewer patients who did not complete were anti-dsDNA-positive and had low $\mathrm{C} 3 / \mathrm{C} 4$ at baseline compared with the 24-month completers (see online supplementary file 5 ).

\section{Clinical outcome measures \\ Overall clinical response}

According to physician assessment, at month 6, 88.4\% and $48.7 \%$ of patients achieved $\geq 20 \%$ and $\geq 50 \%$ improvement in overall clinical response to belimumab, respectively, relative to baseline (figure $2 \mathrm{~A}$ ). Continued improvements were observed at subsequent time intervals with $54.9 \%$ and $32.1 \%$ of patients demonstrating $\geq 20 \%$ and $\geq 50 \%$ relative interval improvement, respectively, between 18 and 24 months.

\section{SLE severity}

At baseline, $77.6 \%$ and $20.2 \%$ of patients had moderate and severe disease, respectively; this reduced to $47.7 \%$ and $2.4 \%$, respectively, at month 6 , and $33.1 \%$ and $1.9 \%$, respectively, at month 24 (figure 2B). A similar pattern of results was seen among the 24-month completer population (see online supplementary file $6 \mathrm{~A})$.

\section{SELENA-SLEDAI}

Mean (SD) SELENA-SLEDAI scores reduced from 12.4 (3.62) at baseline to $5.9(3.22)$ at month $6(\mathrm{n}=122)$. The lower score was maintained at months 12, 18 and 24 (figure 3A).

A similar pattern of results was seen among the 24-month completer population (see online supplementary file $6 \mathrm{~B})$. 
Figure 1 Patient disposition.

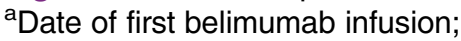
${ }^{b}$ data reported up to month 6 for all enrolled patients; ' affirmatively discontinued belimumab; multiple reasons for discontinuation permitted; ${ }^{\mathrm{d}}$ Case report form (CRF) form not returned; therefore, last observation carried forward for intent-to-treat (ITT) population. AE, adverse event.

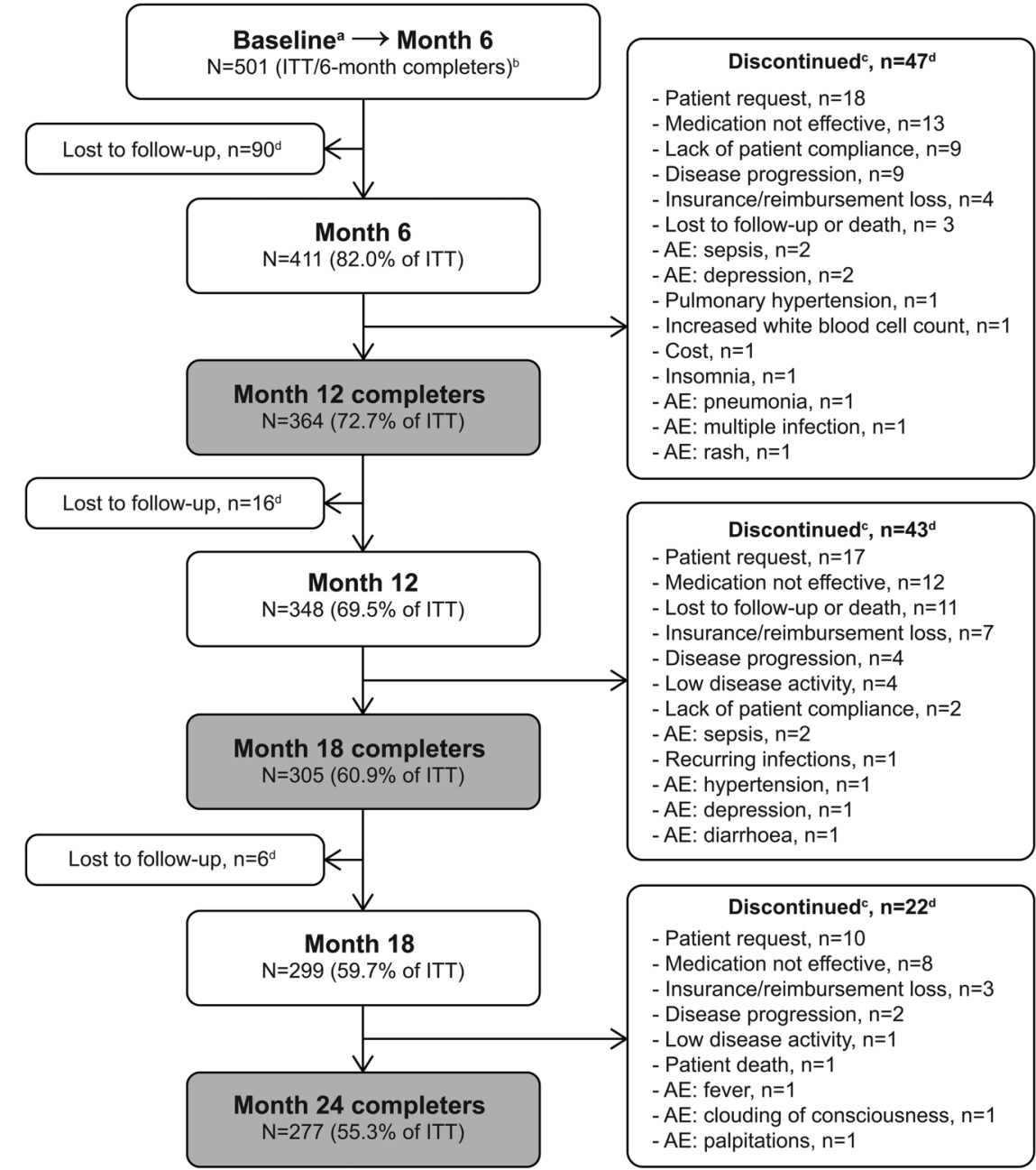

\section{Clinical manifestations}

All patients had at least one moderate/severe clinical manifestation at baseline, as defined by the patient CRF. The incidence of moderate/severe clinical manifestations decreased for all organ domains from baseline to month 24 (figure 3B).

\section{Steroid dose}

At baseline, $386(77.0 \%)$ patients received steroids with a mean (SD) prednisone-equivalent dose of 19.9 (14.39) $\mathrm{mg} /$ day. Of these patients, at month $6,9.1 \%$ had discontinued steroids, $76.9 \%$ were receiving a reduced dose, $11.9 \%$ had no change in dose and $2.1 \%$ had a dose increase; the mean (SD) dose reduced to 8.4 (7.35) $\mathrm{mg} /$ day (figure 3G). The percentage of patients prescribed steroids and the dose received continued to reduce from month 6 to month 24 (mean (SD) dose, 6.1 (9.31) $\mathrm{mg} /$ day). A similar pattern of results was seen among the 24-month completer population (see online supplementary file 6C).

The proportion of all patients receiving $>7.5 \mathrm{mg} /$ day steroids reduced from $67.5 \%$ at baseline to $30.9 \%$ at month $6,21.2 \%$ at month $12,21.6 \%$ at month 18 and $18.4 \%$ at month 24 .

\section{Laboratory parameters}

Laboratory tests remained stable throughout the study; however, among patients with abnormal levels at baseline, the mean levels improved (except total protein in $24 \mathrm{~h}$ urine sample, where patient numbers are very low) (table 2). Improvements were generally greatest from baseline to month 6 and were maintained or continued throughout the study. Among patients categorised as normal at baseline, mean levels remained normal throughout the study.

There was also a reduction in anti-dsDNA-positive patients from $69.1 \%(\mathrm{n}=253 / 366)$ at baseline to $63.0 \%$ $(\mathrm{n}=153 / 243), 50.9 \% \quad(\mathrm{n}=138 / 271)$ and $48.6 \% \quad(\mathrm{n}=139 /$ 286) at months 6,12 and 24 , respectively.

\section{Flare prevention and maintenance of disease control} (24-month completers)

Of 251 patients with a $\geq 20 \%$ improvement in disease between months 0 and 6, 249 (99.2\%) reported no disease flare (worsening of disease) at months 12, 18 and 24. Similarly, of 134 patients with a $\geq 50 \%$ improvement in disease between months 0 and $6,133(99.3 \%)$ reported no worsening of disease at later timepoints. At baseline, 272 patients had moderate or severe disease; of 


\begin{tabular}{|c|c|c|}
\hline & $\begin{array}{l}\text { ITT population } \\
(\mathrm{n}=501)\end{array}$ & $\begin{array}{l}\text { 24-month } \\
\text { completers } \\
(\mathrm{n}=277)\end{array}$ \\
\hline Mean (SD) age, years & $41.3(12.1)$ & $40.9(12.0)$ \\
\hline Gender: female, $\mathrm{n}(\%)$ & $446(89.0)$ & $251(90.6)$ \\
\hline \multicolumn{3}{|l|}{ Race/ethnicity, n (\%) } \\
\hline Caucasian & $265(52.9)$ & $146(52.7)$ \\
\hline African-American & $123(24.6)$ & 69 (24.9) \\
\hline Hispanic & $88(17.6)$ & $43(15.5)$ \\
\hline Asian & $21(4.2)$ & $15(5.4)$ \\
\hline Native American & $4(0.8)$ & 4 (1.4) \\
\hline \multicolumn{3}{|l|}{ Time since SLE diagnosis (years), $\mathrm{n}(\%)$} \\
\hline$<1$ & $17(3.4)$ & $6(2.2)$ \\
\hline $1-5$ & $262(52.3)$ & $156(56.3)$ \\
\hline $6-10$ & $122(24.4)$ & 59 (21.3) \\
\hline$>10$ & $100(20.0)$ & $56(20.2)$ \\
\hline \multicolumn{3}{|l|}{ Severity of SLE at baseline, $\mathrm{n}(\%)^{\star}$} \\
\hline Mild & $9(2.2)$ & $5(1.8)$ \\
\hline Moderate & $319(77.6)$ & $213(76.9)$ \\
\hline Severe & $83(20.2)$ & 59 (21.3) \\
\hline Patients with SELENA-SLEDAI score at baseline, $\mathrm{n}(\%)$ & $122(24.4)$ & $85(30.7)$ \\
\hline Mean (SD) SELENA-SLEDAI score† & $12.4(3.6)$ & $12.3(3.2)$ \\
\hline Median (range) $\ddagger$ & $13.0(2.0-19.0)$ & $12.0(2.0-18.0)$ \\
\hline Patients with SELENA-SLEDAI >10, $n(\%) \dagger$ & $86(70.5)$ & $60(70.6)$ \\
\hline Anti-dsDNA positive and low $\mathrm{C} 3 / \mathrm{C} 4, \mathrm{n}(\%)$ & $252(50.3)$ & $153(55.2)$ \\
\hline \multicolumn{3}{|c|}{ Most common reasons reported by ( $\geq 10 \%$ of patients in either group) for initiating belimumab, $n(\%) \ddagger$} \\
\hline Previous treatment regimen ineffective & $357(71.3)$ & $195(70.4)$ \\
\hline Decrease use of steroids & $286(57.1)$ & $162(58.5)$ \\
\hline Worsening patient condition & $284(56.7)$ & $153(55.2)$ \\
\hline Previous treatment regimen not well tolerated & $127(25.3)$ & $85(30.7)$ \\
\hline Patient request & $54(10.8)$ & $37(13.4)$ \\
\hline \multicolumn{3}{|l|}{ Concomitant SLE medications, $\mathrm{n}(\%)$} \\
\hline Oral steroids & $386(77.0)$ & $218(78.7)$ \\
\hline Antimalarials & $349(69.7)$ & $190(68.6)$ \\
\hline $\begin{array}{l}\text { Immunosuppressants (methotrexate, mycophenolate mofetil, azathioprine, cyclosporine, } \\
\text { cyclophosphamide) }\end{array}$ & $295(58.9)$ & $167(60.3)$ \\
\hline NSAIDs & $82(16.4)$ & $54(19.5)$ \\
\hline Mean (SD) prednisone equivalent dose, mg/day§ & $19.9(14.4)$ & $18.0(12.2)$ \\
\hline Patients receiving prednisone equivalent dose $>7.5 \mathrm{mg} / \mathrm{day}, \mathrm{n}(\%) \S$ & $338(67.5)$ & $190(68.6)$ \\
\hline \multicolumn{3}{|l|}{ SLE organ system manifestation by group, $n(\%) \emptyset$} \\
\hline Musculoskeletal & $316(76.9)$ & $215(77.6)$ \\
\hline Mucocutaneous & 261 (63.5) & 176 (63.5) \\
\hline Constitutional & $233(56.7)$ & $166(59.9)$ \\
\hline Immunological & $222(54.0)$ & $149(53.8)$ \\
\hline Haematological & $145(35.3)$ & $88(31.8)$ \\
\hline Renal & 79 (19.2) & $53(19.1)$ \\
\hline Cardiopulmonary & 68 (16.5) & $46(16.6)$ \\
\hline CNS & 63 (15.3) & 46 (16.6) \\
\hline Vasculitis & $14(3.4)$ & $7(2.5)$ \\
\hline \multicolumn{3}{|c|}{$\begin{array}{l}\text { High disease subgroups are shown in bold. } \\
\text { *SLE severity was assessed based on physician assessment, } n=411 \text { for ITT. } \\
\text { †Among patients with data at baseline. } \\
\text { †Multiple reasons permitted. } \\
\text { §Among patients who received steroids at baseline. } \\
\text { १Reported in > } 1 \% \text { of patients; a patient may have more than one type of clinical manifestation within an organ domain, } n=411 \text { for ITT. } \\
\text { CNS, central nervous system; ITT, intent-to-treat; NSAID, non-steroidal anti-inflammatory drug; SELENA-SLEDAI, Safety of Estrogens in } \\
\text { Lupus National Assessment-Systemic Lupus Erythematosus Disease Activity Index; SLE, systemic lupus erythematosus. }\end{array}$} \\
\hline
\end{tabular}

these, $139(51.1 \%)$ achieved mild disease at month 6, $121(87.1 \%)$ of whom maintained mild disease at all subsequent timepoints. There were 27 patients with
SELENA-SLEDAI $\geq 6$ at baseline that reduced to $<6$ at month 6; of these patients, none had an increase in SELENA-SLEDAI of $>3$ at months 12, 18 or 24 . 
A Overall clinical response

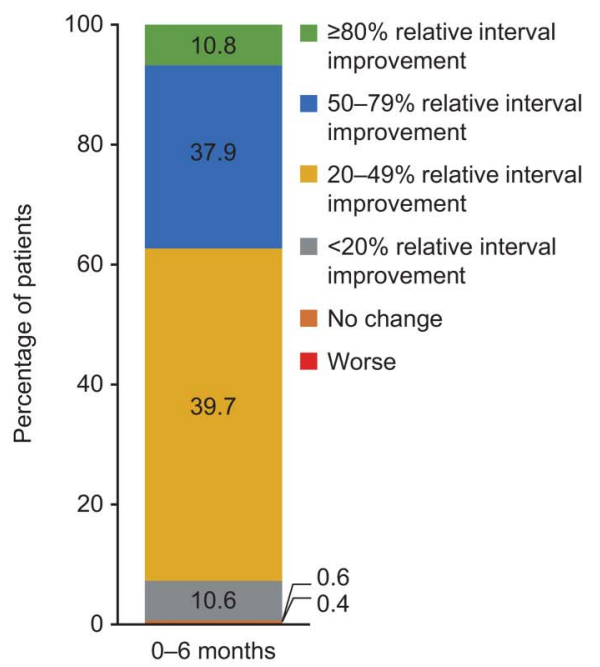

B Disease severity

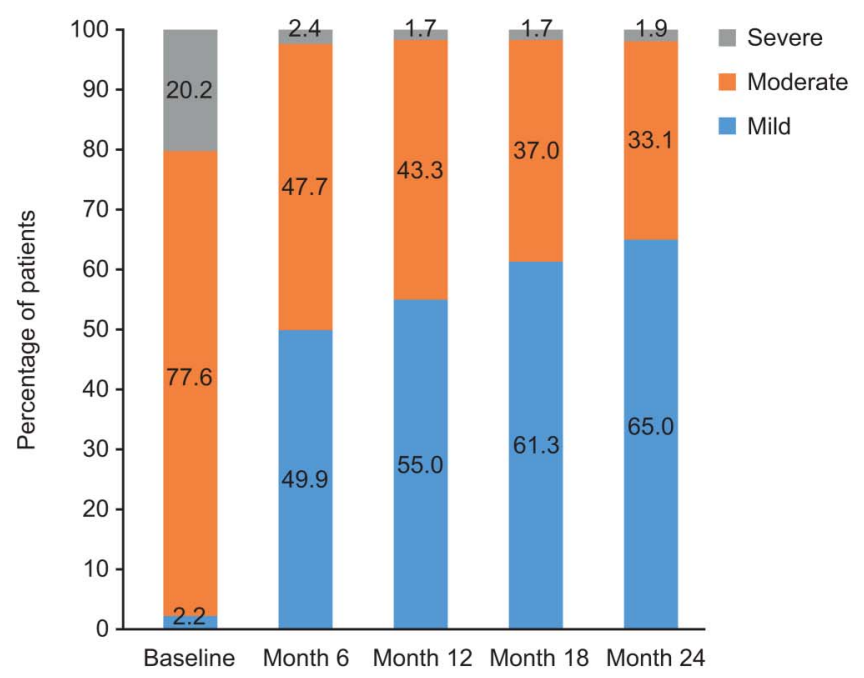

Figure 2 (A) Overall clinical response to belimumab from baseline to 6 months according to physician assessment ( $N=501$, ITT). (B) Disease severity according to physician assessment ( $\mathrm{N}=411$ at each timepoint, intent-to-treat last observation carried forward (ITT LOCF)).

Clinical outcomes among patients with high disease activity at baseline

At baseline, 252 (50.3\%) patients were anti-dsDNA-positive and had low C3/C4, 86/122 (70.5\%) patients with available SELENA-SLEDAI data scored $>10$ and 338 (67.5\%) patients received a steroid dose $>7.5 \mathrm{mg} /$ day (table 1) and were, therefore, classified as having high disease activity. Improvements in clinical response and disease severity in the three high disease subgroups reflected those of the complete population. From baseline to month 6, 128 $(50.8 \%)$ patients in the anti-dsDNA-positive and low C3/ C4 subgroup, 41 (47.7\%) patients in the SELENA-SLEDAI $>10$ subgroup and $174(51.5 \%)$ patients in the steroid dose $>7.5 \mathrm{mg}$ /day subgroup had $\geq 50 \%$ improvement in overall clinical response. Continued relative interval improvements of $\geq 20 \%$ were reported for the majority of patients in each subgroup for all subsequent time periods (data not shown).

At baseline, almost all high disease activity patients had moderate/severe disease according to physician assessment; at month 6, this had reduced to $105(49.6 \%)$ patients in the anti-dsDNA-positive and low C3/C4 subgroup, $52(60.4 \%)$ patients in the SELENA-SLEDAI $>10$ subgroup and $143 \quad(51.5 \%)$ patients in the steroid dose $>7.5 \mathrm{mg} /$ day subgroup; reductions continued or were maintained to month 24 (data not shown).

At baseline, mean (SD) SELENA-SLEDAI score in the SELENA-SLEDAI $>10$ subgroup $(\mathrm{n}=86)$ was $14.3(2.12)$; this decreased to $6.8(3.25)$ at month 6 and 5.6 (3.89) at month 24. Among the patients anti-dsDNA-positive and low C3/C4 with scores available $(\mathrm{n}=63)$, mean $(\mathrm{SD})$ SELENA-SLEDAI decreased from 13.2 (3.36) at baseline to 6.5 (3.32) at month 6 and 5.1 (3.75) at month 24. Among patients receiving a steroid dose $>7.5 \mathrm{mg}$ /day at baseline with scores available $(\mathrm{n}=97)$, mean (SD) SELENA-SLEDAI decreased from 13.0 (3.32) at baseline to 6.1 (3.28) at month 6 and 5.3 (3.73) at month 24 .

Reductions in mean steroid dose were achieved by all three high disease subgroups. Among the subgroup receiving $>7.5 \mathrm{mg} /$ day steroids at baseline, the mean (SD) dose at baseline was $22.0 \quad(14.20) \mathrm{mg} /$ day; this reduced to $9.1(7.58) \mathrm{mg} /$ day at month 6 and 6.4 (9.44) $\mathrm{mg} /$ day at month 24. Among patients in the anti-dsDNA-positive and low $\mathrm{C} 3 / \mathrm{C} 4$ subgroup receiving steroids at baseline $(\mathrm{n}=202)$, the mean $(\mathrm{SD})$ steroid dose at baseline was 20.0 (13.01) $\mathrm{mg} /$ day; this reduced to 8.4 $(7.07) \mathrm{mg} /$ day at month 6 and $7.0(11.04) \mathrm{mg} /$ day at month 24. Among patients in the SELENA-SLEDAI $>10$ subgroup that were receiving steroids at baseline $(n=79)$, the mean (SD) steroid dose at baseline was 24.6 (15.83) $\mathrm{mg} /$ day; this reduced to $9.6(7.86) \mathrm{mg} /$ day at month 6 and $6.8(11.09) \mathrm{mg} /$ day at month 24 .

\section{Comparison of clinical outcomes by ethnicity}

The baseline characteristics of the Caucasian $(n=265)$, African-American $(n=123)$, Hispanic $(n=88)$ and other $(n=25)$ subgroups were similar except that more African-American, Hispanic and other patients had severe disease at baseline compared with Caucasian patients $(28.2 \%, 25.0 \%$ and $32.0 \%$ vs $13.5 \%$, respectively).

For all ethnic subgroups across each 6-month period, the majority of patients were judged to have a $\geq 20 \%$ relative interval improvement in clinical response. The change in overall clinical response at 0-6 months was not statistically significantly different between the ethnic subgroups $(\mathrm{p}=0.2622)$; among the Caucasian subgroup, $124(46.8 \%)$ patients reported $\geq 50 \%$ improvement in clinical response compared with 56 (45.5\%) 

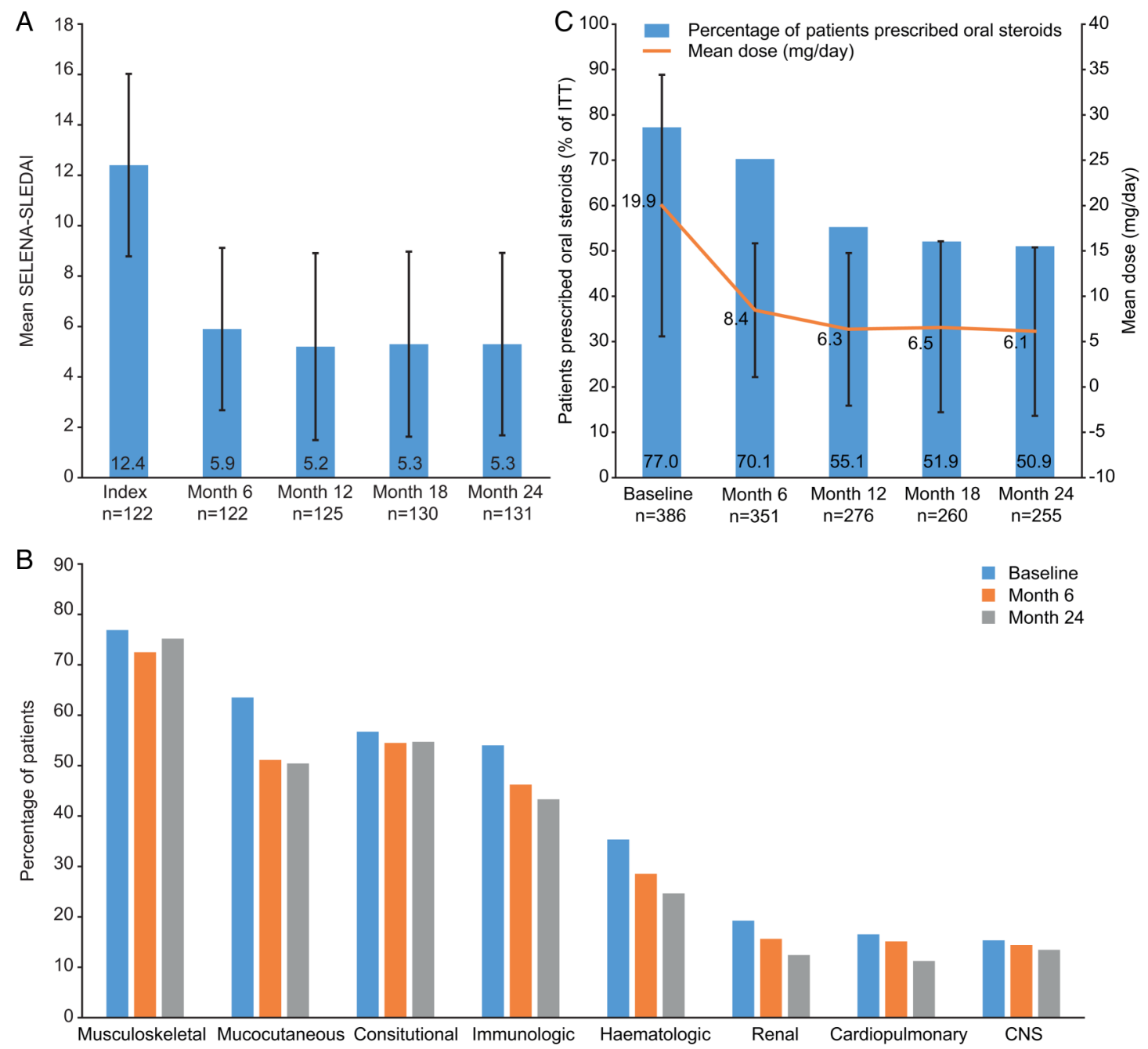

Figure 3 (A) Safety of Estrogens in Lupus National Assessment-Systemic Lupus Erythematosus Disease Activity Index (SELENA-SLEDAI); (B) clinical manifestations (by organ domain) reported by $>5 \%$ intent-to-treat (ITT); (C) Steroid use among patients prescribed oral steroids at baseline. CNS, central nervous system.

African-Americans. At 18-24 months, the proportion of patients reporting $\geq 50 \%$ relative interval improvement in clinical response remained similar among Caucasians $(\mathrm{n}=74,27.9 \%)$ and African-Americans $(\mathrm{n}=33,26.8 \%)$, but was higher among Hispanic $(\mathrm{n}=43,48.9 \%)$ and other $(\mathrm{n}=11,44.0 \%)$ patients $(\mathrm{p}=0.0008)$.

The shift towards milder disease occurred in a similar manner among Caucasian and African-American patients, with 208 (96.8\%), $108(50.2 \%)$ and $74(34.4 \%)$ Caucasians recording moderate/severe disease at baseline, month 6 and month 24, respectively, compared with $102(99.1 \%), 49(47.6 \%)$ and $39(37.9 \%)$ African-Americans, respectively, at these timepoints. Mean (SD) SELENA-SLEDAI scores also reduced similarly for both subgroups; for Caucasians the reduction was from $12.6(3.33)$ at baseline $(\mathrm{n}=72)$ to $5.8(3.19)$ at month $6(\mathrm{n}=72)$ and $5.5(3.95)$ at month $24(\mathrm{n}=80)$, and for African-Americans the reduction was from 12.5 (4.43) at baseline $(\mathrm{n}=22)$ to 6.4 (3.62) at month 6 $(\mathrm{n}=22)$ and $5.7(3.01)$ at month $24(\mathrm{n}=23)$.
Patterns of steroid use were similar between Caucasians and African-Americans. At baseline, 201 (75.8\%) Caucasians compared with 98 (79.7\%) African-Americans were receiving steroids, with a mean (SD) dose of 18.2 (12.54) mg/day and 21.6 (15.46) mg/ day, respectively. Among patients receiving steroids at baseline, there was a decrease in the number receiving steroids and the mean dose. At month 6, 183 (91.0\%) Caucasians were receiving a mean (SD) dose of 8.1 (7.56) $\mathrm{mg}$ /day; at month 24, this had reduced to 131 $(65.2 \%)$ receiving a mean $(\mathrm{SD})$ dose of $5.6(8.15) \mathrm{mg} /$ day; at month $6,89(90.8 \%)$ African-Americans were receiving a mean $(\mathrm{SD})$ dose of $8.9(7.31) \mathrm{mg} /$ day; and at month 24 , this had reduced to $68(69.4 \%)$ receiving a mean (SD) dose of 7.1 (11.24) $\mathrm{mg} /$ day.

\section{HCRU (24-month completer population)}

During the 6 months pre-baseline, $59.6 \%$ of patients had $\geq 1$ unscheduled office visit to their rheumatologist; this decreased to $33.9 \%$ between baseline and month 6 and 
Table 2 Laboratory test results, ITT population

\begin{tabular}{|c|c|c|c|c|c|}
\hline Category (normal cut-off value) & $\begin{array}{l}\text { Baseline, } \\
\text { mean (SD) }\end{array}$ & $\begin{array}{l}\text { Month 6, } \\
\text { mean (SD) }\end{array}$ & $\begin{array}{l}\text { Month 12, } \\
\text { mean (SD) }\end{array}$ & $\begin{array}{l}\text { Month 24, } \\
\text { mean (SD) }\end{array}$ & $\begin{array}{l}\text { p Value (baseline } \\
\text { vs Month 24) }\end{array}$ \\
\hline \multicolumn{6}{|l|}{ White blood cell count $\left(3.5 \times 10^{9} / \mathrm{L}\right)$} \\
\hline Normal at baseline $\left(\times 10^{9} / \mathrm{L}\right)$ & 6.5 (2.12), n=271 & $6.4(2.02), n=271$ & $6.3(2.08), n=276$ & $6.4(2.14), n=276$ & 0.5173 \\
\hline Abnormal (low) at baseline $\left(\times 10^{9} / \mathrm{L}\right)$ & $2.7(0.51), n=94$ & 3.7 (1.41), n=94 & 4.0 (1.19), $n=94$ & $4.1(1.58), n=94$ & $<0.0001$ \\
\hline \multicolumn{6}{|l|}{ Haemoglobin $($ male $=13.5 \mathrm{~g} / \mathrm{dL}$, female $=12 \mathrm{~g} / \mathrm{dL})$} \\
\hline Normal/high at baseline $(\mathrm{g} / \mathrm{dL})$ & $13.3(0.90), n=171$ & $13.1(1.03), n=171$ & $13.1(0.99), n=176$ & $13.1(1.02), n=176$ & 0.1051 \\
\hline Abnormal (low) at baseline ( $\mathrm{g} / \mathrm{dL}$ ) & $10.9(0.95), n=193$ & $11.4(1.01), n=193$ & $11.6(1.06), n=193$ & $11.6(1.13), n=193$ & $<0.0001$ \\
\hline \multicolumn{6}{|l|}{ Platelet count $\left(150 \times 10^{9} / \mathrm{L}\right)$} \\
\hline Normal/high at baseline $\left(\times 10^{9} / \mathrm{L}\right)$ & 262.5 (80.39), $n=276$ & 252.7 (69.47), $n=276$ & $251.9(68.62), n=280$ & 250.5 (72.11), $n=280$ & 0.0355 \\
\hline Abnormal (low) at baseline $\left(\times 10^{9} / \mathrm{L}\right)$ & $105.0(26.55), n=88$ & 131.8 (41.77), $n=88$ & 145.8 (49.29), $n=88$ & 155.9 (54.04), $n=89$ & $<0.0001$ \\
\hline \multicolumn{6}{|c|}{ ESR $($ male $<50$ years $=15 \mathrm{~mm} / \mathrm{h}$, male $\geq 50$ years and female $<50$ years $=20 \mathrm{~mm} / \mathrm{h}$, female $\geq 50$ years $=30 \mathrm{~mm} / \mathrm{h}$ ) } \\
\hline Normal/high at baseline $(\mathrm{mm} / \mathrm{h})$ & $12.7(7.44), n=56$ & $13.9(9.68), n=56$ & 12.5 (10.04), n=60 & 10.0 (6.65), $n=60$ & 0.0225 \\
\hline Abnormal (elevated) at baseline $(\mathrm{mm} / \mathrm{h})$ & $48.7(20.98), n=273$ & $32.1(17.4), n=273$ & $26.4(16.86), n=277$ & 25.0 (17.56), $n=280$ & $<0.0001$ \\
\hline \multicolumn{6}{|c|}{ Serum creatinine (male $=1.3 \mathrm{mg} / \mathrm{dL}$, female $=1.1 \mathrm{mg} / \mathrm{dL}$ ) } \\
\hline Normal/low at baseline (mg/dL) & $0.8(0.18), n=284$ & $0.8(0.19), n=284$ & $0.8(0.18), n=291$ & $0.9(0.22), n=296$ & 0.6660 \\
\hline Abnormal (high) at baseline (mg/dL) & $1.6(0.63), n=56$ & $1.4(0.53), n=56$ & $1.3(0.47), n=59$ & $1.3(0.48), n=59$ & $<0.0001$ \\
\hline \multicolumn{6}{|l|}{ Spot urine protein-creatinine ratio $(0.2 \mathrm{mg} / \mathrm{mg})$} \\
\hline Normal at baseline $(\mathrm{mg} / \mathrm{mg})$ & $0.1(0.04), n=40$ & $0.1(0.04), n=40$ & $0.1(0.05), n=41$ & $0.1(0.14), n=46$ & 0.1880 \\
\hline Abnormal (high/very high) at baseline $(\mathrm{mg} / \mathrm{mg})$ & $0.8(0.78), n=41$ & $0.7(0.65), n=41$ & $0.6(0.71), n=53$ & $0.5(0.53), n=58$ & $<0.0001$ \\
\hline \multicolumn{6}{|c|}{$24 \mathrm{~h}$ urine collection (normal to high $300 \mathrm{mg} / 24 \mathrm{~h}$, high to very high $500 \mathrm{mg} / 24 \mathrm{~h}$ ) } \\
\hline Normal at baseline $(\mathrm{mg} / 24 \mathrm{~h})$ & $283.0(0), n=1$ & $257.0(0), n=1$ & $209.0(0), n=1$ & 207 (132.28), n=3 & 0.7500 \\
\hline Abnormal (high) at baseline (mg/24 h) & 417.8 (61.9), $n=5$ & 450.0 (119.79), $n=5$ & 714.3 (454.96), $\mathrm{n}=6$ & 688.3 (477.47), $n=6$ & 0.3125 \\
\hline Abnormal (very high) at baseline $(\mathrm{mg} / 24 \mathrm{~h})$ & 1014.0 (466.9), $n=4$ & $839.5(225.7), n=4$ & $1643.8(1344.2), n=5$ & 1466.9 (1242.3), $n=7$ & 1.0000 \\
\hline \multicolumn{6}{|l|}{ CRP (10 mg/L) } \\
\hline Normal at baseline (mg/L) & $1.8(1.68), n=246$ & $1.0(1.07), n=246$ & $1.0(1.97), n=253$ & $0.8(0.9), n=260$ & 0.0156 \\
\hline Abnormal (elevated) at baseline (mg/L) & $16.2(4.77), n=7$ & 7.6 (4.42), $n=7$ & $5.3(7.34), n=7$ & $5.3(8.45), n=7$ & $<0.0001$ \\
\hline \multicolumn{6}{|l|}{ AST (male=20 IU/L, female=36 IU/L) } \\
\hline Normal/low at baseline (IU/L) & $21.8(6.60), n=243$ & $23(9.46), n=243$ & $23.2(9.93), n=248$ & $23.8(9.55), n=248$ & 0.0025 \\
\hline Abnormal (high) at baseline (IU/L) & $43.9(17.26), n=56$ & 32.6 (13.76), $n=56$ & $33.2(16.77), n=59$ & $31.2(16.06), n=60$ & $<0.0001$ \\
\hline \multicolumn{6}{|l|}{ ALT (male $=45$ IU $/ L$, female $=33$ IU/L) } \\
\hline Normal/low at baseline (IU/L) & $20.6(6.70), n=236$ & $21.3(7.93), n=236$ & $21.6(9.23), n=244$ & $22.3(8.69), n=246$ & 0.0159 \\
\hline Abnormal (high) at baseline (IU/L) & 46.9 (15.44), $n=70$ & $36.6(17.31), n=70$ & 35 (18.13), n=72 & $33.2(17.71), n=73$ & $<0.0001$ \\
\hline \multicolumn{6}{|l|}{ C3 complement ( $75 \mathrm{mg} / \mathrm{dL})$} \\
\hline Normal/high at baseline (mg/dL) & 114.4 (30.03), $n=142$ & 119.1 (28.98), $n=142$ & 120.1 (27.90), $n=148$ & 118.7 (28.68), $n=150$ & 0.0244 \\
\hline Abnormal (low) at baseline (mg/dL) & $53.3(16.68), n=136$ & $70.3(25.39), n=136$ & $80.2(28.01), n=142$ & $82.2(32.21), n=145$ & $<0.0001$ \\
\hline \multicolumn{6}{|c|}{ C4 complement $($ male $=12 \mathrm{mg} / \mathrm{dL}$, female $=13 \mathrm{mg} / \mathrm{dL})$} \\
\hline Normal/high at baseline $(\mathrm{mg} / \mathrm{dL})$ & $25.5(13.54), n=122$ & 26.9 (13.03), $n=122$ & 26.7 (11.94), $n=128$ & $27.6(12.08), n=130$ & 0.0012 \\
\hline Abnormal (low) at baseline (mg/dL) & $7.4(3.04), n=156$ & $12.8(7.46), \mathrm{n}=156$ & $14.5(7.60), \mathrm{n}=162$ & $16.2(9.20), n=165$ & $<0.0001$ \\
\hline
\end{tabular}


$28.9 \%$ between month 18 and month 24 . The mean number of unscheduled office rheumatologist visits among all patients decreased from 1.2 in the 6 months prior to belimumab initiation to 0.6 baseline-month 6 and at months $18-24$. From the 6 -month period prebaseline to the 6 -month period post-baseline, the percentages of patients with $\geq 1$ ER visit $(15.2-6.1 \%), \geq 1$ hospitalisation (4.7-0.4\%) and those receiving ancillary care decreased (19.1-7.9\%); over the same time period, the mean number of ER visits (0.2 to 0.1$)$ and hospitalisations (0.1 to 0.0$)$ also decreased. HCRU remained lower at all timepoints post-baseline compared with the 6-month period pre-baseline, except for the number of scheduled physician visits, which increased from 3.9 in the 6-month period pre-baseline to 4.1 in the 6-month period post-baseline.

HCRU was consistent across the ethnic subgroups with the exception that both pre-baseline and during months 0-6 African-American patients had a higher mean number of hospitalisations, number of ER visits and number of days spent in hospital compared with the other ethnic subgroups (data not shown).

\section{DISCUSSION}

This observational study examined the clinical effectiveness of marketed belimumab plus SoC in patients with SLE in US clinical practice. Over the 24-month period, patients had an overall improvement in disease activity according to physician assessment of clinical response and disease severity. Furthermore, the number of patients receiving steroids and the mean dose of steroids decreased and patients with abnormal laboratory values associated with active SLE improved. Generally, the improvements were more pronounced in the first six months of treatment; however, improvements continued or were maintained through 24 months. HCRU decreased over the study period, with fewer unscheduled rheumatologist visits, ER visits and hospitalisations reported. Subgroup analyses demonstrated that patients with high disease activity and African-Americans responded to belimumab in a similar manner to the overall population. As the results from the phase 3 studies with belimumab in the African-American population were inconclusive, ${ }^{2}$ the observed positive outcomes in this subgroup provide valuable information.

Observational studies enable data collection from clinical practices across a wide geographic area and provide a more realistic reflection of treatment within clinical practice compared with clinical trials where stricter inclusion/exclusion criteria, dosing limitations and inflexible concomitant medication management can limit the generalisability of data to clinical practice.

The study design has some limitations. The lack of a control group limits the conclusions that can be made as pre-treatment and post-treatment measurements cannot account for the natural course of this highly variable disease. It is important to understand patient attrition in longitudinal analyses. Approximately half of patients did not complete the study; furthermore, the ITT population may have been enriched with patients who were more likely to respond to belimumab as only patients who received belimumab for a minimum of 6 months were included; patients who discontinued treatment early may have done so due to a lack of response or for safety and tolerability reasons. ITT analyses with the LOCF method were employed to account for missing values, but still may not provide a true picture as patients have their status artificially stabilised (favourably or unfavourably) at the point of attrition. The LOCF method assumes that attrition is not related to patient characteristics or outcomes. Analyses of the 24-month completer population show the same trends in results as the ITT population, suggesting that employing the LOCF method has not substantially affected the results.

In the BLISS-52 trial, clinically meaningful overall improvements were seen with belimumab $10 \mathrm{mg} / \mathrm{kg}$ plus SoC as early as week 16; improvements in Physician Global Assessment score were significant at week 24, and an association between belimumab and reduction in disease flare were observed. ${ }^{3}$ In this study, we also report improvements in disease severity within 6 months of initiating belimumab and the improvements were largely maintained without worsening disease throughout the study, suggesting prevention of flares.

Steroid use reduction is an important factor in treatment of SLE as prolonged use of high-dose steroids is a major cause of long-term damage and morbidity. ${ }^{6} 7$ In agreement with the BLISS studies, we have defined $>7.5 \mathrm{mg} /$ day as high dose. Post hoc analyses of the BLISS trials suggested a steroid-sparing effect of belimumab among patients with serologically active disease. ${ }^{8}$ This study also suggests that belimumab has a steroidsparing effect (among all patients and those with high disease activity), with a reduction in both the number of patients receiving steroids and the mean dose. However, it should be noted that the reasons for steroid reduction/discontinuation were not provided and may have been due to reasons other than improved disease, (eg, side effects, patient preference); nevertheless, the steroid data provide an objective measure of disease state.

Renal involvement, experienced by up to $60 \%$ of patients with SLE, is a common source of morbidity. ${ }^{9}$ Although belimumab is not indicated for patients with severe lupus nephritis, a pooled post hoc analysis of the BLISS trials suggested that patients with renal involvement who received belimumab plus SoC had fewer renal flares (defined by increases in proteinuria) compared with patients receiving placebo. ${ }^{10}$ The present study supports this finding as the spot urine protein:creatinine ratio and serum creatinine remained stable, and for patients with abnormal values, these parameters improved at 6 months and continued to gradually improve through 24 months. 
Other laboratory test results generally demonstrated that patients who had normal levels at baseline remained normal and patients who had abnormal levels at baseline improved towards normal levels, including C3, C4, white blood cell count, platelet count, haemoglobin, CRP and ESR. In addition, the proportion of patients with positive anti-dsDNA decreased over the 24 months. These objective data suggest improvements in patients' disease state throughout the study, although without a placebo-control group this cannot be conclusively determined to be due to belimumab treatment.

This study was not designed to assess safety and tolerability. The BLISS- $52^{3}$ and BLISS- $76^{4}$ trials demonstrated an acceptable safety profile for belimumab and ongoing randomised control studies and large observational studies will provide further postmarketing data on safety and tolerability (eg, NCT01705977 $7^{11}$ and NCT01729455 ${ }^{12}$ ).

There is no standard disease assessment tool for SLE recommended by rheumatology professional organisations such as the American College of Rheumatology (ACR) or the European League against Rheumatism; therefore, the primary outcome of this study was physician-assessed relative interval improvement in overall clinical response. This was designed to mimic the ACR response assessment used in rheumatoid arthritis. In this study, we found that no validated disease assessment tool was routinely used in non-academic clinical practice as nearly $50 \%$ of physicians never use any tool and SELENA-SLEDAI, the most commonly used validated tool, was only used by $14.1 \%$ of physicians. The lack of any consistently used validated tool is, therefore, a general challenge when assessing SLE treatment. We have used multiple methods of assessing disease severity; improvements were observed in all measures, giving confidence in these findings.

As patient numbers were small for SELENA-SLEDAI, conclusions should be made with caution. Limited data were also available for some of the laboratory tests; nevertheless, these do provide some objective data. In addition, a limitation of the CRFs is that they were an interpretation of what was written in the charts. Furthermore, under-reporting may have occurred if not all events were reported to the physician or recorded on the patient chart.

In conclusion, the findings of this observational study provide evidence of reduced disease activity in patients with SLE who received at least 6 months of belimumab plus SoC in a clinical practice setting. In the first six months of belimumab therapy, according to physician assessment, SLE severity decreased and patients continued to improve or maintained their improvement throughout 24 months. In addition, belimumab added to SoC demonstrated reduction in SELENA-SLEDAI, flare prevention and maintenance of disease control. These favourable outcomes were achieved in the setting of objective reductions in steroid use, improvements in laboratory parameters and lower HCRU. These results from clinical practice support the findings of randomised clinical trials that have demonstrated the efficacy of belimumab.

\section{Author affiliations}

${ }^{1}$ MedStar Washington Hospital Center, Washington DC, USA

${ }^{2}$ University of California, San Francisco, California, USA

${ }^{3}$ GlaxoSmithKline, Research Triangle Park, North Carolina, USA

${ }^{4}$ Medical Data Analytics, Parsippany, New Jersey, USA

${ }^{5}$ GlaxoSmithKline, Philadelphia, Pennsylvania, USA

${ }^{6}$ GlaxoSmithKline, Middlesex, Uxbridge, UK

Acknowledgement Medical writing assistance was provided by Katie White, $\mathrm{PhD}$, of Fishawack Indicia, funded by GlaxoSmithKline.

Contributors All authors made substantial contributions to the conception or design of the work; or the acquisition, analysis or interpretation of the data. All authors contributed to drafting and revising this manuscript, have approved the final version and are accountable for the accuracy and integrity of the work.

Funding Human Genome Sciences and GlaxoSmithKline sponsored this study and were involved in the conception, design, implementation, study supervision, data analysis and interpretation, manuscript drafting, revision and approval.

Competing interests CEC and MD'E are paid consultants for GlaxoSmithKline. CMa is an employee of Medical Data Analytics, who developed the study design and carried out data collection (funded by GlaxoSmithKline). HK is an employee of and holds stock in GlaxoSmithKline. CMo, VK and DJC were employees of GlaxoSmithKline at the time of the study and hold stock in GlaxoSmithKline.

Patient consent Exemption was provided for obtaining patient consent as the study was non-interventional and data were de-identified.

Ethics approval The study was approved by the New England Institutional Review Board and was conducted in accordance with the Declaration of Helsinki 2008 and the International Society for Pharmacoeconomics and Outcomes Research guidelines.

Provenance and peer review Not commissioned; externally peer reviewed.

Data sharing statement No additional data are available.

Open Access This is an Open Access article distributed in accordance with the Creative Commons Attribution Non Commercial (CC BY-NC 4.0) license, which permits others to distribute, remix, adapt, build upon this work noncommercially, and license their derivative works on different terms, provided the original work is properly cited and the use is non-commercial. See: http:// creativecommons.org/licenses/by-nc/4.0/

\section{REFERENCES}

1. Baker KP, Edwards BM, Main SH, et al. Generation and characterization of LymphoStat-B, a human monoclonal antibody that antagonizes the bioactivities of B lymphocyte stimulator. Arthritis Rheum 2003;48:3253-65.

2. GlaxoSmithKline. Belimumab prescribing information. http://us.gsk com/products/assets/us_benlysta.pdf (accessed Jul 2015).

3. Navarra SV, Guzmán RM, Gallacher AE, et al. Efficacy and safety of belimumab in patients with active systemic lupus erythematosus: a randomised, placebo-controlled, phase 3 trial. Lancet 2011;377:721-31.

4. Furie R, Petri M, Zamani O, et al. A phase III, randomized, placebo-controlled study of belimumab, a monoclonal antibody that inhibits B lymphocyte stimulator, in patients with systemic lupus erythematosus. Arthrits Rheum 2011;63:3918-30.

5. Ray WA, Griffin MR, Avorn J. Evaluating drugs after their approval for clinical use. N Engl J Med 1993;329:2029-32.

6. Chatham WW, Kimberly RP. Treatment of lupus with corticosteroids. Lupus 2001;10:140-7.

7. Zonana-Nacach A, Barr SG, Magder LS, et al. Damage in systemic lupus erythematosus and its association with corticosteroids. Arthritis Rheum 2000;43:1801-8. 
8. van Vollenhoven RF, Petri MA, Cervera R, et al. Belimumab in the treatment of systemic lupus erythematosus: high disease activity predictors of response. Ann Rheum Dis 2012;71:1343-9.

9. Cameron JS. Lupus nephritis. J Am Soc Nephrol 1999;10: 413-24.

10. Dooley M, Houssiau F, Aranow C, et al. Effect of belimumab treatment on renal outcomes: results from the phase 3 belimumab clinical trials in patients with SLE. Lupus 2013;

22:63-72.

11. GlaxoSmithKline. Belimumab assessment of safety in SLE (BASE). Study synopsis. https://clinicaltrials.gov/ct2/show/NCT01705977

(accessed Jul 2015).

12. GlaxoSmithKline. Safety and effectiveness of belimumab in system lupus erythematosus registry (SABLE). Study synopsis. https:// clinicaltrials.gov/ct2/show/NCT01729455 (accessed Jul 2015). 